\title{
Etat des lieux de l'élevage caprin en milieu paysan au Togo
}

\author{
Atouga Yembliman Djagba ${ }^{1 *}$ Bèdibètè Bonfoh ${ }^{1}$ \\ Habrè Bassowa ${ }^{1}$ Kodjo Aklikokou ${ }^{2}$ N'gonlbi Kanour ${ }^{1}$
}

\begin{abstract}
Mot-clés
Caprins, chevreau, Djallonké, productivité, Togo
\end{abstract}

Submitted: 8 August 2018

Accepted: 13 November 2019

Published: 24 February 2020

DOI: $10.19182 /$ remvt.31840

\section{Résumé}

Les caprins constituent au Togo la seconde espèce d'élevage après les volailles en terme d'effectif. La disponibilité de sa viande reste pourtant rare. L'Etat togolais, à travers des projets de développement, a initié la recapitalisation du cheptel national d'animaux à cycle court. L'objectif de l'étude était la connaissance des faiblesses des pratiques d'élevage des caprins au Togo en vue de proposer des axes de recherche pour son amélioration et son développement. Des enquêtes ont été menées auprès de 129 chefs de ménages des deux sexes. Les éleveurs étaient des paysans, des salariés ou des retraités. Les enquêtes ont couvert l'ensemble du pays, prenant en compte sa diversité pluviométrique et végétale. Des données sur les pratiques d'élevage, les soins vétérinaires, les maladies et les autres contraintes ont été recueillies. Les résultats ont montré que $69 \%$ des éleveurs étaient instruits, $91 \%$ étaient mariés, et $26 \%$ des propriétaires étaient des femmes. La main d'œuvre familiale jouait un rôle important. Les activités liées à l'élevage étaient confiées aux femmes et aux enfants. La taille moyenne du cheptel par ménage était de 18 caprins. Les chèvres adultes constituaient $44 \%$ de l'effectif contre $2 \%$ de mâles adultes entiers. Le taux de productivité au sevrage était de 0,54 chevreau par mère. Les animaux disposaient d'un abri ; dans $85 \%$ des élevages, ils recevaient périodiquement une complémentation alimentaire et dans $27 \%$ d'entre eux un complément minéral à base de sel de cuisine. Les soins vétérinaires ont été observés dans $64 \%$ des élevages. Cette étude a permis de révéler quelques faiblesses relatives aux soins vétérinaires, à l'utilisation de géniteurs améliorés et à la disponibilité d'abris. Des formations sur les itinéraires techniques de l'élevage des caprins sont nécessaires pour améliorer leurs performances.

- Comment citer cet article: Djagba A.Y., Bonfoh B., Bassowa H., Aklikokou K., Kanour N., 2020. Assessment of goat rearing in the farming environment in Togo. Rev. Elev. Med. Vet. Pays Trop., 73 (1): 11-19, doi: 10.19182/remvt.31840

\section{INTRODUCTION}

Aujourd'hui, à travers le monde, l'élevage de chèvres occupe de plus en plus de place dans les économies nationales car il constitue un facteur de développement économique, notamment dans les zones rurales (Chetroiu et al., 2013). Selon la FAO, la planète comptait 1002810368 chèvres en 2016, le continent asiatique 556019726 (55,5\%), et le continent africain 387667193 (38,7\%) (Faostat, 2017). Par ailleurs, la population caprine, représentant $43,5 \%$ des ruminants domestiques, a connu un croît annuel au cours de la dernière décennie de $3,4 \%$, contre $3,2 \%$ chez les ovins et 4,2 \% chez les bovins (Missohou et al., 2016). Cette croissance montre que l'élevage des chèvres a

\footnotetext{
1. Institut togolais de recherche agronomique, BP 01 Anié, Togo.

2. Université de Lomé, Togo.

* Auteur pour la correspondance

Email : atouga16@yahoo.fr
}

généralement une tendance ascendante, à la fois en termes de stocks et de niveau de production (Chetroiu et al., 2013). Longtemps, l'élevage des caprins n'a pas bénéficié des appuis des programmes de développement du cheptel par rapport aux autres espèces (Monfort, 1990).

Au Togo, l'élevage des caprins est pratiqué sur tout le pays et, avec 3401512 têtes, cette espèce est la deuxième en effectif, derrière les volailles (29 159000 têtes) (Faostat, 2017). Contrairement à d'autres régions du monde où la chèvre n'est pas élevée pour sa viande qui est peu appréciée (Vanwarbeck, 2008), la viande caprine est très appréciée et consommée par la majorité de la population en Afrique, en particulier celle du bouc Djallonké castré au sud du Togo, où elle est au menu des restaurants. Les caprins sont sacrifiés lors des funérailles, des mariages, des sacrifices, des fêtes de fin d'année ou des baptêmes, qualifiés de prélèvements sociaux (Gnanda et al., 2016 ; Barry, 1985 ; Okello, 1985 ; Tchouamo et al., 2005). Ils contribuent fortement à l'économie familiale, à la durabilité des moyens de subsistance et à la réduction de la pauvreté (Saidu et al., 2006 ; Yandia, 2012 ; Gnanda et al., 2016). Chez certains éleveurs, c'est le capital de départ pour la constitution d'un troupeau de 
bovins (Gnanda et al., 2016). Ils constituent une épargne et tiennent un rôle important dans la notoriété de l'éleveur (Zakara, 1985). Ils peuvent être élevés dans une gamme étendue de conditions agroenvironnementales et selon un large éventail de systèmes de production (Alexandre et al., 2012). Certains éleveurs partagent parfois l'abri avec les animaux. Ces derniers bénéficient rarement d'un apport de supplément alimentaire et de soins sanitaires en raison principalement de l'absence de personnel technique ou du coût des traitements (Barry, 1985). Les conditions climatiques, à l'instar d'autres facteurs comme les maladies, les conflits sociopolitiques, régissent les effectifs des animaux des différentes zones agroécologiques, éléments structurants des systèmes d'élevage en termes de répartition des espèces et des races, de capacité de charge, de pressions sanitaires et de productivité individuelle (Otte et Chilonda, 2002). La majorité des caprins élevés au Togo sont de la race Djallonké. En raison de sa trypanotolérance cette race est élevée dans toutes les régions du pays. Malgré cette importance, il existe peu d'éleveurs professionnels de caprins avec de grands effectifs, à l'instar des élevages de volailles, d'ovins ou de bovins au Togo. L'élément caractéristique principal de cet élevage est le manque d'une chaîne de valeur ou d'activités de transformation réelles qui permettraient d'augmenter les revenus des éleveurs à l'instar de celui des producteurs de lait dans d'autres pays. Plusieurs raisons sont souvent évoquées par les acteurs de la filière. Certaines contraintes, comme l'alimentation, la santé, la gestion du troupeau, le manque d'organisation structurelle et institutionnelle, sont désignées comme freinant le développement de la filière de l'élevage des petits ruminants (Wasso et al., 2018).

Le Togo, pays côtier de l'Afrique de l'Ouest, à l'instar de la Côte d'Ivoire, connaît depuis quelques décennies une diminution de la consommation de la viande de caprins au profit de celle de bovins. Cette situation peut être due à la préférence alimentaire, au coût, à l'adaptabilité du canal de distribution et surtout à la rareté des caprins en quantité et de bon format sur le marché (Montfort, 1990). Dans la politique nationale de développement de l'élevage par la recapitalisation du cheptel, le Gouvernement togolais avait décidé de relancer l'élevage des petits ruminants et des volailles par la diffusion de géniteurs améliorés. Le besoin estimé pour les petits ruminants était de 5000 géniteurs, dont au moins 2000 boucs. A la fin de trois années de campagne (2013-2015), les géniteurs diffusés étaient de 2341 béliers et 289 boucs. Le faible effectif de boucs était dû au manque de disponibilité chez les éleveurs et au niveau du centre de sélection.

Plusieurs projets d'appui au développement de l'élevage caprin ont été menés, mais les résultats sont restés en deçà des attentes des gouvernements et des organisations non gouvernementales (ONG). Cet élevage reste peu performant en termes de production, conduisant l'Etat, dans l'exécution du Projet d'appui au secteur agricole (PASA), à faire la recapitalisation du cheptel national par la diffusion de géniteurs améliorateurs. Les principales causes de ce manque de décollage sont la baisse de la motivation des éleveurs vers la fin des projets, l'absence de relève pour la continuité des activités à la suite de la disparition du bénéficiaire du projet, et aussi le manque d'organisation professionnelle dans cet élevage en vue des négociations et de la défense de leurs intérêts (Montfort, 1990).

Avec une bonne prolificité, atteignant $185 \%$ (Missohou et al., 2016), reconnue pour la chèvre Djallonké, il est souvent signalé de fortes mortalités, d'où les faibles effectifs rencontrés chez les éleveurs. Le système d'élevage des caprins au Togo semble être orienté vers la production de viande. Défini comme un ensemble d'éléments en interaction, organisés par l'homme dans le cadre d'une activité d'élevage visant à obtenir des productions variées (par exemple lait, viande, cuirs et peaux, travail, fumure) ou à atteindre tout autre objectif (Landais et Bonnemaire, 1996), le système d'élevage caprin pratiqué au Togo est très diversifié selon les régions. Compte tenu de la nécessité de sortir le monde agricole de sa précarité, les politiques actuelles visent à transformer l'économie de subsistance dont l'élevage en un secteur pourvoyeur de ressources et d'emplois. Ainsi, un état des lieux de l'élevage des caprins dans les différentes zones agroécologiques du pays a été fait afin de permettre aux éleveurs de connaître les faiblesses de leurs pratiques, aux chercheurs d'orienter les axes de recherche, et aux autorités de bien connaître les défis de cet élevage et de mettre les moyens pour les relever.

\section{MATERIEL ET METHODES}

\section{Sites de l'étude}

Cette enquête a couvert la majorité du territoire national à travers 18 préfectures. Les différentes zones agroécologiques (figure 1) sont caractérisées par la pluviométrie annuelle, la végétation et le relief. Les caractéristiques des différentes zones agroécologiques sont : a) la zone du littoral (région Maritime) caractérisée par un climat côtier frais et une végétation moins dense ; b) la zone de forêt mésophile (Plateaux Ouest), zone humide de l'Atakora caractérisée par des précipitations abondantes, une strate de sous-bois et des herbes à larges feuilles ; c) la zone de savane humide (Plateaux Est), abondamment arrosée, arborée, constituée de l'ensemble du Dahoméen dans le bassin du Mono ; d) la zone de l'Atakora (région Centrale) couvrant partiellement la zone des massifs de collines avec une strate herbacée composée de graminées en touffe (Andropogon sp., Hyparrhenia sp.) ; e) la zone des massifs de collines ou zone de forêt sèche (région Kara), strate herbacée composée de graminées en touffes; et f) la zone soudanienne (région des Savanes), caractérisée par la présence du baobab (Adansonia digitata), d'arbres épineux et d'une strate herbacée constituée entre autres d'Aristida sp., de Chloris sp., et d'Hyparrhenia sp.

La forte pluviométrie et la densité de la végétation favorisent la pullulation des glossines (tsé-tsé) Glossina morsitans morsitans

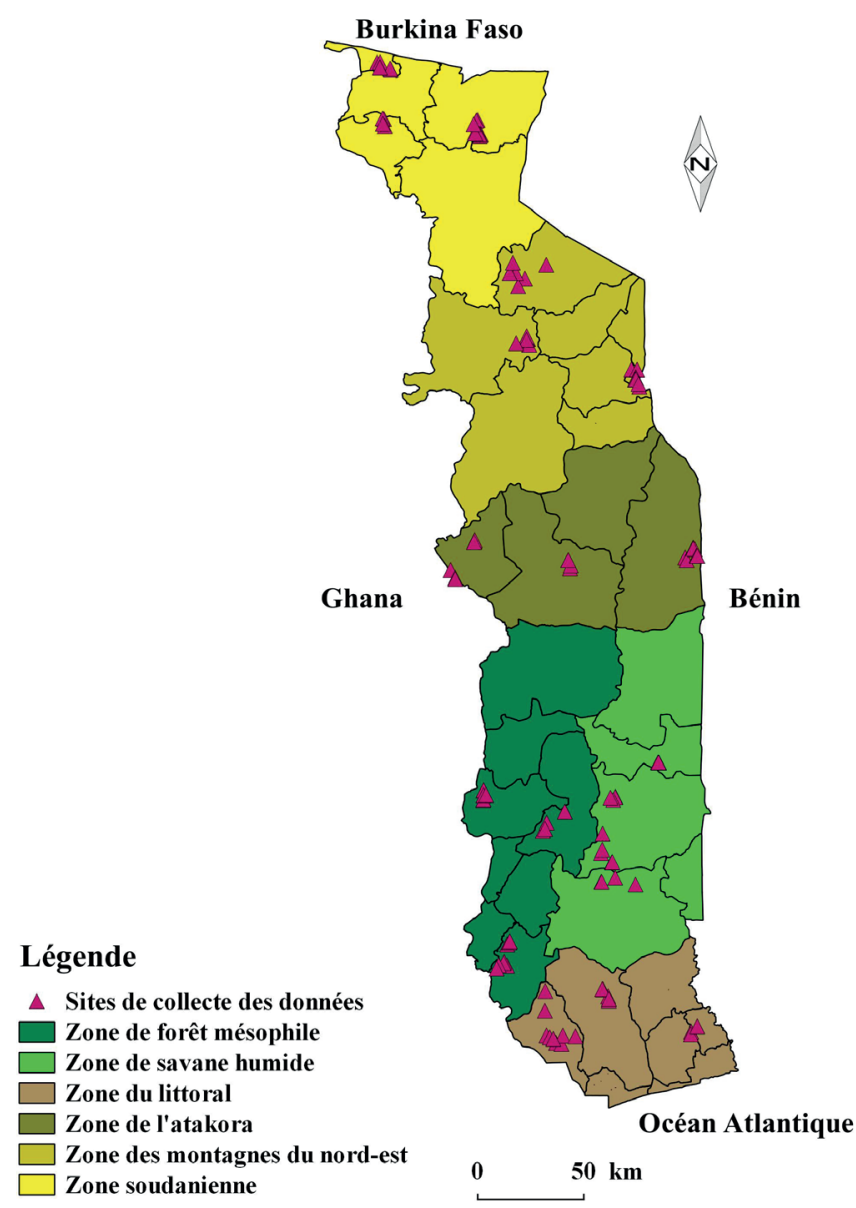

Figure 1 : carte du Togo montrant les zones écologiques et les sites d'élevage caprin de l'enquête. 
(Wiedemann, 1850) et G. palpalis gambiensis (Vanderplank, 1911), dans certaines zones (Plateaux Ouest, Plateaux Est et région Centrale). L'élevage de toutes les espèces de ruminants, notamment des caprins non trypanotolérants, reste limité.

\section{Echantillonnage et recueil des données}

Des entretiens avec les chefs de ménage ou toute personne propriétaire de caprins, hommes ou femmes, ont été menés auprès de 129 ménages à travers toutes les régions du Togo, avec un accès aux animaux et aux infrastructures. Les éleveurs ont été sélectionnés avec l'appui des techniciens supérieurs d'élevage chargés de l'appuiconseil ou de certains auxiliaires villageois d'élevage (formés sur le tas par les vétérinaires), chargés des soins vétérinaires. La distance minimale entre deux ménages était d'un kilomètre.

Une fiche d'enquête a servi pour le recueil des données lors de l'entretien avec les éleveurs et pendant la visite des parcs et du milieu. Cette fiche était semi-ouverte ; les éleveurs fournissaient les données qualitatives ou quantitatives (entre autres système d'élevage, type de complément alimentaire, composition de la famille, situation civile et niveau d'instruction) alors que les données sur l'effectif du troupeau étaient collectées par observation. La constitution de chaque troupeau, l'état des abris ou du matériel d'élevage (abreuvoirs, mangeoires) étaient évalués par observation directe dans les parcs, sur les pâturages, et par la consultation des cahiers de soins vétérinaires. Des questions ouvertes ont permis de recueillir les informations dans les groupes de discussion (le plus souvent improvisés) organisés au niveau de certaines agences de vulgarisation, ou en tirant parti des réunions qui se tenaient chez des chefs de villages le jour de l'enquête.

\section{Description de l'échantillon}

Les éleveurs n'étaient pas exclusivement éleveurs de caprins, certains élevaient aussi d'autres espèces comme les volailles (poules ou pintades), les ovins, les porcs ou les bovins. Ils cultivaient du maïs, du mil, du sorgho, de l'igname, du manioc, de l'arachide, du soja, du niébé ou du coton. Aucun élevage caprin ne produisait de lait de chèvre, les animaux étaient destinés à la boucherie. Dans la majorité des cas les caprins étaient considérés comme une épargne qui permettait de satisfaire les besoins urgents (par exemple frais de santé, maladie, cérémonie, scolarisation, mariage) par la vente. La plupart des éleveurs n'avaient pas de compte d'épargne et donc n'avaient pas accès aux crédits (prêts) au niveau des microfinances. La main d'œuvre était familiale dans la plupart des foyers pour l'élevage et les travaux des champs. Le recours à la main d'œuvre temporaire consistait davantage en des travaux de métayage pour les labours et le sarclage des champs chez ceux qui en avaient les moyens (retraités, fonctionnaires ou commerçants). Les hommes étaient responsables du labour des champs, et les femmes et les enfants du sarclage. Les femmes étaient aussi chargées du semis, des travaux de ménage, et de veiller à l'alimentation des animaux avec l'appui des personnes âgées de la maison ou de leurs enfants. Les animaux étaient vendus soit aux commerçants soit à d'autres éleveurs, ou directement aux bouchers à la ferme ou au marché.

\section{Analyses statistiques des données}

Les données collectées ont été traitées sous tableur Excel version 2013. Les analyses en tableau de contingence ont été effectuées avec le logiciel SPSS version 20. Afin de déterminer les relations de dépendance et leur intensité entre a) les variables présence de parc, application des soins vétérinaires et disponibilité d'un bouc géniteur, et les régions, b) les variables distribution des compléments alimentaires et minéraux, et âge de l'éleveur, et c) la disponibilité d'un bouc géniteur, et l'effectif du troupeau, les tests du $\chi^{2}$ et de $\mathrm{V}$ de Cramer ont été effectués avec le même logiciel. Les graphiques montrant les tendances (secteurs et histogrammes) de certains paramètres (répartition des éleveurs, niveau d'étude, effectif des troupeaux, pratique de soins et distribution de compléments) ont été tracés avec Excel.

\section{RESULTATS}

\section{Situation de famille et niveau d'étude des éleveurs}

La majorité des éleveurs étaient mariés (117 sur 129 ; $91 \%$ ) et $7 \%$ étaient veuves. La majorité des propriétaires déclarés des chèvres étaient des adultes et 34 (26\%) étaient des femmes.

Sur l'ensemble des régions, $32 \%$ des éleveurs ont déclaré n'avoir pas été scolarisés, $12 \%$ avoir fait des études supérieures (université ou formations professionnelles) et $56 \%$ avoir un niveau d'instruction intermédiaire (niveau maximum le collège), certains d'entre eux ayant bénéficié du programme d'alphabétisation. Les éleveurs dans cet échantillon ayant fait des études supérieures étaient des retraités ou des fonctionnaires affectés. Le niveau d'instruction a varié selon les régions ; le taux d'éleveurs n'ayant pas été scolarisés était supérieur à $45 \%$ dans les régions Savanes, Centrale et Plateaux Est. Par ailleurs les femmes étaient moins scolarisées que les hommes. Plus de $59 \%$ d'entre elles n'étaient pas instruites contre $23 \%$ des hommes, seulement $6 \%$ d'entre elles avaient fait des études supérieures contre $14 \%$ des hommes, et $35 \%$ avaient un niveau d'instruction intermédiaire contre $63 \%$ des hommes.

\section{Taille du troupeau, sexe-ratio dans les élevages et gestion reproductive}

La race dominante était la race Naine de l'Afrique de l'Ouest (West African Dwarf goat) (2350 têtes), communément appelée race Djallonké. Elle était présente dans tout le pays. La Sahélienne (29 têtes), la deuxième race la plus présente, était localisée dans la région des Savanes et dans les zones périurbaines. Enfin, la Rousse de Maradi (11 têtes) était surtout rencontrée dans les zones périurbaines.

La taille moyenne du troupeau dans les élevages était de 18 chèvres. Parmi les éleveurs, $25 \%$ avaient un effectif caprin inférieur à 10 têtes, $62 \%$ un effectif de 10 à moins 30 têtes, et $13 \%$ au minimum 30 têtes.

Le tableau I montre que la majorité des effectifs était constituée de femelles : $44 \%$ de chèvres adultes, $16 \%$ de chevrettes sevrées (âgées de 5 à 10 mois) et $14 \%$ de chevrettes sous la mère (moins de 4 mois). Pour les mâles, cette proportion était de moins de $2 \%$ pour les mâles entiers adultes, $14 \%$ pour les jeunes mâles sevrés de moins de 12 mois et $10 \%$ pour les chevreaux mâles sous la mère. Au total, $83 \%$ des élevages ne disposaient pas de boucs adultes entiers.

Le tableau I montre un effectif faible de chevreaux mâles et femelles sous la mère (562 chevreaux) par rapport à celui des chèvres mères (1041 mères). Le ratio était de 0,54 chevreau pour une chèvre mère. Les mouvements démographiques au sein des troupeaux n'ont pas

\section{Tableau I}

Répartition des animaux selon l'âge et le sexe dans les régions du Togo

\begin{tabular}{lrrrrrr} 
Région & Chevrette & Jeune & Adulte & Chevreau & Jeune & Bouc \\
Maritime & 55 & 36 & 136 & 34 & 53 & 8 \\
Plateau Ouest & 68 & 68 & 152 & 41 & 57 & 5 \\
Plateau Est & 58 & 78 & 189 & 35 & 61 & 9 \\
Centrale & 61 & 68 & 191 & 48 & 60 & 11 \\
Kara & 32 & 69 & 172 & 49 & 63 & 5 \\
Savanes & 46 & 55 & 201 & 35 & 35 & 6 \\
Effectif & 320 & 374 & 1041 & 242 & 329 & 44 \\
\hline \% Total & 13,6 & 15,9 & 44,3 & 10,3 & 14,0 & 1,9
\end{tabular}


été enregistrés. En effet, de fortes mortalités des chevreaux avant le sevrage ont été signalées verbalement par les éleveurs et les aides vétérinaires locaux. L'absence de cahier de bergerie n'a pas permis de voir les périodes de mise bas, de mortalités, d'achat, de vente ou de perte des animaux. L'identification individuelle des animaux n'a pas été constatée dans ces élevages.

Le problème des géniteurs dans les élevages a été constaté surtout chez les éleveurs possédant un petit effectif. Les résultats de l'analyse statistique ont montré une différence significative pour la présence de boucs selon les régions (tableau II) mais aussi selon l'effectif du troupeau (tableau III). L'exploitation précoce des boucs dans certaines régions par la castration ou la vente afin d'éviter le vol a été souvent signalée. De plus, la présence de boucs géniteurs distribués par le gouvernement a expliqué la présence de ces animaux dans certaines régions. Parmi les critères de sélection des éleveurs était la disponibilité de géniteur bouc adulte, les éleveurs ayant un effectif faible ( $<10$ têtes) ne disposaient pas de géniteurs boucs adultes. Ceux réservés comme géniteurs constituaient moins de $2 \%$ de l'effectif total des animaux. Le sexe-ratio boucs adultes / chèvres mères était de 1:24. Pour l'ensemble des animaux enregistrés, le pourcentage des mâles (chevreaux sous la mère, jeunes mâles de moins d'un an, boucs castrés et boucs entiers de plus d'un an) était de 26,2\%. Les chèvres étaient saillies la plupart du temps après la période des cultures pendant la divagation par des boucs d'origine inconnue. La faible présence de géniteurs dans les élevages s'expliquait par le fait que les éleveurs ayant un petit effectif étaient souvent des éleveurs vulnérables : ils castraient les mâles nés dans leur élevage afin d'augmenter leur valeur marchande et d'éviter leur perte par vol. Certaines chèvres étaient saillies par des jeunes boucs ( $<1$ an d'âge) car dans ces élevages la castration des jeunes boucs se faisait selon les propriétaires deux à trois mois après le sevrage. D'autres éleveurs estimaient qu'avec la présence des jeunes boucs les accouplements des mères étaient assurés. Cette pratique a pour conséquence l'accouplement des chèvres avec leurs propres chevreaux ou avec des boucs d'origine inconnue lors de la divagation. Selon les éleveurs, le faible poids des chevreaux à la naissance était dû aux mises bas en saison pluvieuse et à la consanguinité liée à la monte des chèvres par leurs chevreaux.

\section{Types d'abri des caprins}

Chez $89 \%$ des ménages visités, il existait un abri pour les animaux. Les abris étaient améliorés ou modernes chez $27 \%$ d'entre eux (figure 2). Avec l'appui des projets, $7 \%$ des éleveurs (surtout ceux ayant un effectif moyen) ont bénéficié de chèvreries de type moderne. Cet abri était constitué d'un mur de ciment, d'une cour entourée de grillage et était équipé de matériel de bergerie (abreuvoirs et mangeoires). Les éleveurs âgés disposaient de moins d'abris modernes pour les animaux que les jeunes. Concernant le type d'abri, la réparation ou reconstruction à chaque fin de saison des chèvreries ou des bergeries traditionnelles a été signalée par les éleveurs. Les principales causes étaient les intempéries (vent, pluie, attaque par les termites) en raison de la précarité des matériaux utilisés (terre battue et paille). Certains éleveurs ont pu installer des fosses fumières et y mettre les déchets des animaux après balayage afin d'épandre le fumier au début de la saison des pluies.

Il y avait une différence significative pour la présence de parc pour animaux selon la région (tableau III). Dans les régions Savanes et Plateaux Ouest, il existait des abris pour les caprins alors que son absence atteignait $30 \%$ dans les régions Kara et Centrale (tableau I). La majorité des chèvreries étaient de type traditionnel, en terre battue couverte de paille, avec des palmes, de vieilles tôles ou des bâches en guise de toit. La plupart des chèvreries (80 \%) étaient construites par les éleveurs euxmêmes avec des matériaux locaux pour protéger les animaux contre la pluie ou les voleurs. Cependant, ces abris étaient très rarement balayés sauf au moment de faire l'évacuation des poudrettes à l'approche de la saison des cultures, favorisant la prolifération des puces notée par plusieurs éleveurs.

Les observations ont montré que plus l'éleveur avait un niveau d'étude élevé plus le type d'abri était amélioré (figure 2). Parmi

\section{Tableau III}

Analyses statistiques pour la présence de bouc améliorateur selon I'effectif du troupeau caprin au Togo

\begin{tabular}{lcc}
\hline Effectif & \multicolumn{1}{c}{ Bouc améliorateur } \\
& Absent (\%) & Présent (\%) \\
\hline Faible & 100 & 0,0 \\
Moyen & 80,5 & 19,5 \\
Grand & 85,0 & 15,0 \\
\hline$\chi^{2}$ de Pearson & & \\
Probabilité & & \\
V de Cramer & & 0,028 \\
\end{tabular}

\section{Tableau II}

Analyses statistiques selon les régions au Togo pour la présence de bouc améliorateur, d'un parc et I'application des soins

\begin{tabular}{|c|c|c|c|c|c|c|c|}
\hline \multirow[t]{2}{*}{ 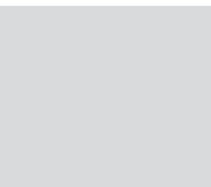 } & \multirow[t]{2}{*}{ Région } & \multicolumn{2}{|c|}{ Bouc améliorateur } & \multicolumn{2}{|c|}{ Parc } & \multicolumn{2}{|c|}{ Soin } \\
\hline & & $\begin{array}{c}\text { Absent } \\
(\%)\end{array}$ & $\begin{array}{c}\text { Présent } \\
(\%)\end{array}$ & $\begin{array}{c}\text { Absent } \\
(\%)\end{array}$ & $\begin{array}{c}\text { Présent } \\
(\%)\end{array}$ & $\begin{array}{c}\text { Absent } \\
(\%)\end{array}$ & $\begin{array}{c}\text { Présent } \\
(\%)\end{array}$ \\
\hline & Centrale & 91,3 & 8,7 & 30,4 & 69,6 & 13,0 & 87,0 \\
\hline & Kara & 100 & 0,0 & 30,4 & 69,6 & 21,7 & 78,3 \\
\hline & Maritime & 65,0 & 35,0 & 10,0 & 90,0 & 70,0 & 30,0 \\
\hline & Plateau Est & 95,5 & 4,5 & 13,6 & 86,4 & 72,7 & 27,3 \\
\hline & Plateau Ouest & 66,7 & 33,3 & 0,0 & 100 & 19,0 & 81,0 \\
\hline & Savanes & 95,0 & 5,0 & 0,0 & 100 & 15,0 & 85,0 \\
\hline$\chi^{2}$ de Pearson & & \multicolumn{2}{|c|}{21,2} & \multicolumn{2}{|c|}{16,5} & \multicolumn{2}{|c|}{37,1} \\
\hline Probabilité & & \multicolumn{2}{|c|}{0,001} & \multicolumn{2}{|c|}{0,006} & \multicolumn{2}{|c|}{0,000} \\
\hline V de Cramer & & \multicolumn{2}{|c|}{0,405} & \multicolumn{2}{|c|}{0,358} & \multicolumn{2}{|c|}{0,536} \\
\hline
\end{tabular}




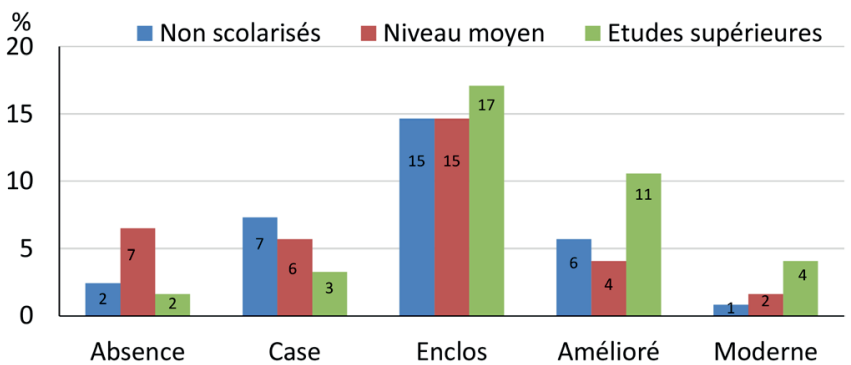

Figure 2 : type d'abri des caprins selon le niveau d'instruction des éleveurs (Togo).

$28 \%$ des abris de caprins améliorés ou modernes, plus de la moitié (53\%) se trouvaient chez les éleveurs ayant fait des études supérieures. Quelques éleveurs de niveau d'instruction moyen (retraités, enseignants d'école, commerçants et infirmiers dans les villages), mettaient aussi en place des abris améliorés pour les animaux. Ils se faisaient assister par des techniciens ou imitaient les plans des bergeries améliorées mises en place dans leur localité ou au niveau du centre de recherche. Dans ces cas, les bergeries étaient des enclos couverts de tôles avec des parcs de nuit dont les murs étaient construits en ciment avec une cour en bois pour servir le fourrage. Dans chaque ménage il n'existait qu'un abri pour l'ensemble des caprins.

\section{Pratique des soins aux animaux}

Sur le plan national, la majorité des éleveurs (64\%) ont affirmé que leurs animaux étaient suivis sur le plan sanitaire par un technicien supérieur d'élevage ou un aide vétérinaire présent dans la localité. L'analyse statistique (tableau II) a montré qu'il y avait une différence significative dans l'apport de soins aux caprins selon les régions. Certaines régions sont reconnues comme des zones d'élevage par excellence (Savanes, Kara et Centrale). Ces régions accueillent davantage de jeunes auxiliaires villageois d'élevage (AVE) formés par les vétérinaires privés et installés dans ces régions pour travailler avec eux. Dans les campagnes des régions Plateaux Est et Maritime, plus de $70 \%$ des élevages n'avaient pas d'accès facile aux services vétérinaires. Les AVE étaient rares et les vétérinaires privés travaillaient plutôt dans leurs cliniques où ils vendaient aussi les médicaments. Leurs déplacements dans les campagnes étaient rares et il a été signalé moins d'engouement des jeunes dans ces localités pour ce service que dans d'autres régions. Les soins étaient en général limités au déparasitage interne et à la vaccination lors des campagnes nationales organisées par le gouvernement. Plusieurs cas de kératoconjonctivites, d'ecthyma et des diarrhées blanches ont été observés chez les chevreaux sous la mère. La gale, les maladies respiratoires (pneumopathies) ont été aussi notées et des foyers de peste des petits ruminants (PPR) signalés par les éleveurs et les vétérinaires lors des campagnes 2013-2015. Ces maladies ont été rencontrées dans toutes les zones agroécologiques mais à des fréquences différentes selon que l'éleveur était suivi ou non par un agent vétérinaire. Cependant, des éleveurs ne faisant pas vacciner leurs animaux ont déclaré avoir été victimes de mortalités d'animaux ou témoins de mortalités d'animaux vaccinés contre la PPR. Certains éleveurs et les chargés du suivi sanitaire des animaux reconnaissaient la régression du format adulte des animaux. Les principales causes de mortalités évoquées étaient la faiblesse de poids des chevreaux à la naissance pour cause de mise bas multiple, l'ecthyma, la kératoconjonctivite, et d'autres maladies banales mais très fréquentes comme les diarrhées (coccidiose ou diarrhées blanches). L'insalubrité des parcs a contribué aussi pour une part non négligeable aux mortalités car les parasites externes, surtout les puces, étaient présents dans plus de $90 \%$ des parcs traditionnels.

\section{Conduite alimentaire du troupeau}

Les principaux modes de gardiennage étaient la claustration partielle (48\%) et l'attache au piquet (47\%) pendant la période des cultures (tableau IV). La différence de gardiennage selon les régions s'expliquait par les habitudes culturales et la pression sur la terre. Plus de $85 \%$ des éleveurs ont affirmé donner une complémentation alimentaire mais seulement $27 \%$ donnaient une complémentation minérale à base de sel de cuisine aux caprins (figure 3 ).

\section{Tableau IV}

Répartition des éleveurs en fonction du mode de conduite alimentaire des caprins au Togo (\% total)

\begin{tabular}{lccccrrr} 
Système d'élevage & Maritime & Plateau Est & Plateau Ouest & Centrale & Kara & Savanes & National \\
\hline Divagation totale & 0 & 3 & 0 & 0 & 0 & 0 & 2,3 \\
Claustration partielle & 16 & 17 & 17 & 12 & 0 & 0 & 48,1 \\
Piquet hivernage & 3 & 2 & 3 & 11 & 22 & 20 & 47,3 \\
Conduite partielle & 1 & 0 & 1 & 1 & 0 & 0 & 2,3
\end{tabular}

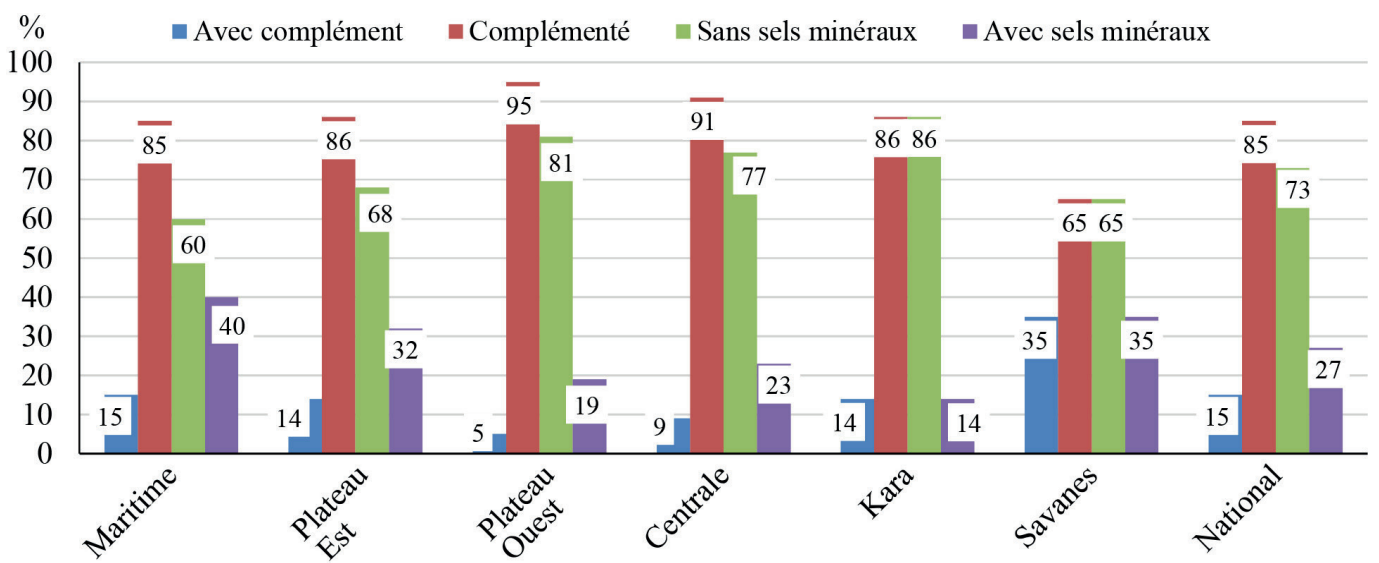

Figure 3 : répartition des éleveurs distribuant des compléments alimentaires et des sels minéraux selon les régions (Togo). 
La conduite des caprins au pâturage à l'instar des ovins était rare ; elle a été observée chez $2 \%$ des éleveurs (tableau IV). Dans ces élevages, les caprins Djallonké dormaient avec les caprins Sahéliens et/ ou les ovins dans le même enclos. Dans la majorité des élevages, les caprins étaient soit mis au piquet (mode le plus pratiqué dans la partie nord du pays, figure 4), soit mis sous système d'affouragement pendant la saison des cultures (figure 5). Les enfants et les femmes se chargeaient de la cueillette des feuilles et des herbes, puis de l'abreuvement. Les caprins se nourrissaient d'herbe ou de feuilles mortes qu'ils trouvaient lors de la divagation. Pendant la saison des cultures, dans les élevages pratiquant la claustration, les caprins étaient nourris avec des branchages, des palmes et des graminées comme Panicum maximum, Pennisetum sp. Un faible niveau d'investissement dans l'alimentation de qualité des animaux (absence de bons compléments alimentaires, rareté de la complémentation minérale à base de pierre à lécher, c'était le sel de cuisine qui était souvent donné) a influencé la productivité. Les animaux recevaient souvent comme complément alimentaire des épluchures de manioc, du son de soja, de maïs ou de sorgho, ou du remoulage. Les déchets de récoltes (fanes d'arachide et de niébé) étaient souvent stockés et distribués aux animaux pendant la saison sèche, période au cours de laquelle le pâturage était maigre et où les caprins ne se contentaient plus des feuilles mortes et des écorces des arbres.

Les enfants allant encore à l'école amenaient les animaux en brousse pour les mettre au piquet le matin et les ramenaient le soir dans les abris. Les femmes âgées (les mères et les veuves surtout) jouaient

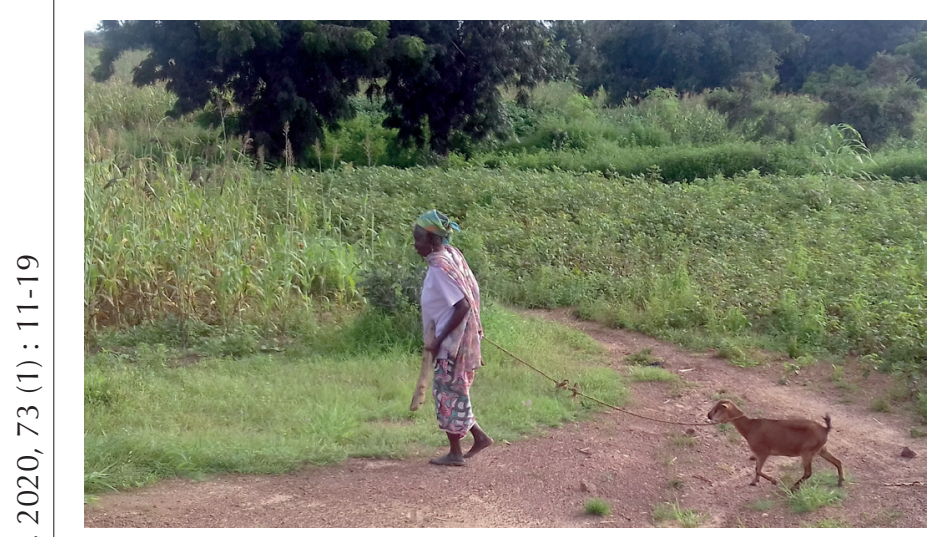

Figure 4 : mise au piquet d'une chèvre au nord du Togo.

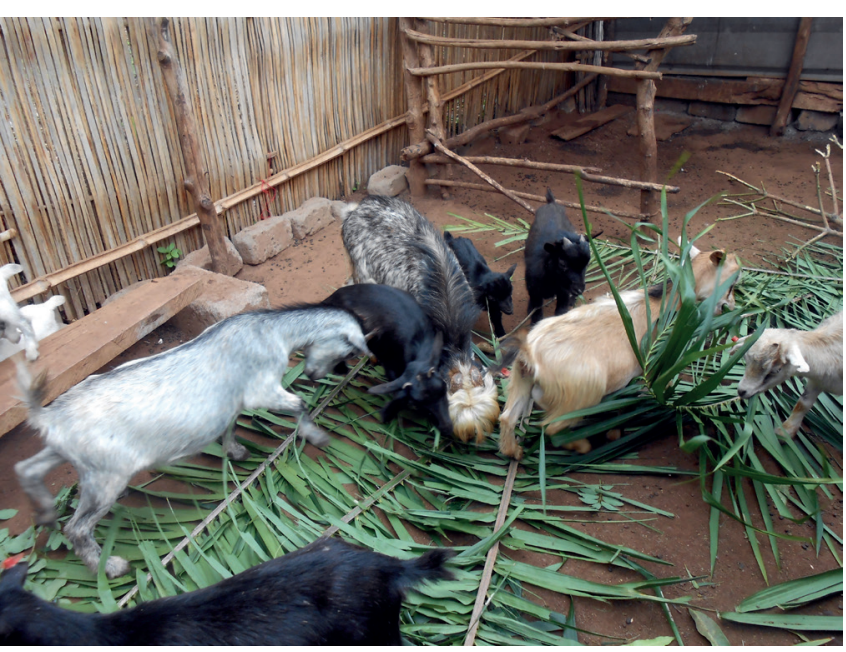

Figure 5 : affouragement des caprins pendant la saison des cultures au Togo. aussi un rôle important dans la surveillance des chèvres. Ces femmes, ménagères n'ayant pas d'activités génératrices de revenus spécifiques, étaient soit transformatrices de sorgho en bière locale, soit transformatrices de manioc en gari, soit de petites commerçantes qui disposaient aussi de petits champs de maïs, de riz, d'arachide ou de niébé. Elles s'occupaient de la mise au piquet des chèvres au cas où les enfants n'avaient pas pu le faire. Dans les situations de claustration, c'étaient toujours les enfants et leurs mères qui étaient chargés de l'affouragement, de l'abreuvement et du nettoyage des parcs. Dans les zones urbaines, les fourrages étaient soit coupés le long des rues ou des rivières, soit achetés au marché ou livrés par des revendeuses au niveau des maisons. Chez les éleveurs non mariés, les animaux étaient sous la surveillance de leur mère ou des jeunes frères et sœurs. La plupart des éleveurs considéraient les caprins comme une source de revenus rapides donc une épargne facilement accessible. Dans les régions des Savanes, l'eau est rare en saison sèche et la chaleur très élevée (pouvant atteindre $45^{\circ} \mathrm{C}$ à l'ombre). Les animaux en divagation pour la recherche de quelques feuilles mortes revenaient à la ferme afin de recevoir quelques fanes d'arachide ou de niébé et boire de l'eau souvent servie dans des bassines, des abreuvoirs de fortune à base de bidons coupés ou de canaris cassés laissés devant la maison. En effet, les chèvres n'avaient pour aliment que les herbes qui à certaines périodes de l'année étaient de mauvaise qualité, voire rares (la quantité d'aliments servis en complément était insuffisante, voire absente par moment).

La complémentation du troupeau a varié selon l'âge de l'éleveur : les personnes plus âgées (plus de 55 ans) donnaient moins de compléments alimentaires que les plus jeunes $\left(\chi^{2}, p=0,04\right.$; tableau $\left.V\right)$. En effet, les jeunes éleveurs ayant suivi des séances de formation théoriques et pratiques organisées par l'Etat ou les ONG sur les bonnes pratiques d'élevage donnaient quelques compléments aux animaux.

\section{Tableau V}

Analyses statistiques selon l'âge de l'éleveur pour la complémentation alimentaire des caprins au Togo

\begin{tabular}{lcc} 
Age des éleveurs & \multicolumn{2}{c}{ Complémentation alimentaire } \\
\cline { 2 - 3 } & Absente (\%) & Présente (\%) \\
\hline Adultes & 9,3 & 90,7 \\
$>55$ ans & 22,2 & 77,8 \\
\hline$\chi^{2}$ de Pearson & 4,153 & \\
Probabilité & 0,042 & \\
V de Cramer & 0,179 & \\
& &
\end{tabular}

La complémentation minérale était rare chez les éleveurs. La plupart de ceux qui ont déclaré donner des minéraux à leurs caprins le faisaient de façon irrégulière. Elle était donnée sous forme de sel de cuisine mélangé ou saupoudré sur les compléments alimentaires (son de tchouk ou gousses de niébé surtout). Les pierres à lécher étaient souvent servies dans les fermes des éleveurs disposant d'un peu de moyens financiers ou dans les élevages dans lesquels il y avait un nombre important d'ovins conduits au pâturage. Les principales raisons évoquées par les éleveurs pour la nondistribution de complément minéral étaient l'ignorance de l'effet bénéfique de la pierre à lécher pour la productivité des animaux, sa nondisponibilité et son coût élevé, surtout dans les localités éloignées des grandes villes. 


\section{DISCUSSION}

Le taux de femmes propriétaires de caprins a été supérieur à celui de $18 \%$ rapporté dans les régions Kara et Savanes au Togo (Guingouain, 2017). L'alimentation, la conduite, l'abreuvement et la surveillance des caprins par les enfants, les ménagères et les personnes plus âgées étaient une pratique généralisée dans la plupart des pays africains (Killanga et Traoré, 1999 ; Najari et Gaddour, 2008 ; Tchouamo et al., 2005 ; Wasso et al., 2018).

Par rapport au niveau d'instruction, la plupart des éleveurs avaient un niveau moyen (niveau du secondaire). Le taux d'éleveurs n'ayant pas fait de longues études était élevé et a confirmé que les activités agricoles étaient pratiquées par la population qui avait un niveau d'instruction moindre (Guingouain, 2017). Les taux d'éleveurs non scolarisés dans les différentes régions ont été inférieurs à celui de $70 \%$ rapporté dans la commune rurale d'Aït Bazza au Maroc (Nassif et al., 2011) et de 62,9\% dans les Hauts-Plateaux de l'Ouest Cameroun (Manjeli et al., 1994). Cependant au Cameroun dans la région Ouest, Tchouamo et al. (2005) ont signalé un taux de $76 \%$ d'éleveurs de caprins instruits. Les femmes propriétaires des caprins de cette étude étaient les moins scolarisées. Cette situation confirme le fait que depuis longtemps en milieu paysan, l'éducation des filles n'est pas une priorité pour les parents. Elles s'occupent bien des animaux pour la mise au piquet ou l'apport d'affouragement et de l'eau, mais elles ignorent l'importance d'une bonne complémentation alimentaire et minérale.

La taille moyenne des effectifs enregistrée dans les élevages a varié de 4 à 60 têtes avec une moyenne de 18 têtes pour l'ensemble des éleveurs. Elle était supérieure à la moyenne de sept chèvres par ménage observée dans les Hauts-Plateaux de l'Ouest Cameroun (Manjeli et al., 1994), et de six chèvres par ménage en République démocratique du Congo (Wasso et al., 2018). Cette taille était cependant inférieure à la moyenne de 55 chèvres par éleveur signalée dans la commune rurale d'Ait Bazza au Maroc (Nassif et al., 2011). Le pourcentage d'éleveurs ayant un effectif supérieur à 10 têtes de caprins a varié selon les régions. Seulement dans les régions Kara et Savanes, plus de $30 \%$ des éleveurs avaient un effectif inférieur à 10 têtes. Cette variation d'effectif selon la zone d'élevage a été signalée au Burkina (Tamboura et Berte, 1994).

Le problème de vol d'animaux, surtout de boucs, est rapporté notamment par Guingouain (2017) au Togo, et Wassao et al. (2018) en RDC. Très peu d'éleveurs de caprins ont bénéficié de l'appui des projets pour les boucs et équipements d'élevage. Ceux ayant un effectif supérieur à 10 têtes ont reçu des appuis avec des boucs améliorateurs donnés par l'Etat et les ONG. En effet, entre 2012 et 2015, l'Etat togolais à travers PASA a procédé à la distribution chez les éleveurs de 2341 béliers et seulement 289 boucs géniteurs. La proportion des chèvres mères enregistrée dans les élevages a été voisine de celle trouvée au Sénégal par Ba Diao et al. (1994). Le taux d'absence de boucs entiers adultes dans les élevages a été supérieur à celui trouvé dans la région Ouest Cameroun (82\%) (Tchouamo et al., 2005).

Le faible taux de productivité des chèvres dans les élevages a été comparable à celui signalé au Cameroun (Manjeli et al., 1994). Il a été inférieur à 0,85 chevreau/chèvre enregistré au Sénégal (Tourrand et Landais, 1996). L'insuffisance de pâturage et la rareté de la complémentation alimentaire des caprins ont constitué un facteur limitant de l'élevage. Cette contrainte est aussi signalée pour les systèmes d'élevage au Sénégal (Tourrand et Landais, 1996). Le faible apport d'aliments complémentaires par les éleveurs a fait dire à certains auteurs que les caprins sont les «parents pauvres » en matière de complémentation alimentaire et de suivi sanitaire par rapport aux ovins et aux volailles, surtout dans les ménages où l'élevage des bovins n'est pas pratiqué (Gnanda et al., 2016).
La proportion des ménages disposant d'un abri pour les caprins dans notre étude a été plus élevée que celle de $66 \%$ signalée au Burkina (Tamboura et Berté, 1994). Dans certains élevages, les caprins ont partagé l'abri le soir avec les ovins ou les bovins utilisés pour la culture attelée. Cette pratique a été observée lors des enquêtes dans la majorité des ménages de la région nord du pays. Le même abri était partagé dans le cas où les effectifs des différentes espèces étaient faibles, généralement moins de dix têtes par espèce. Cette pratique est aussi signalée en Sardaigne (Ligios et al., 2004). Dans certains cas, les chèvres étaient attachées au piquet au niveau du paturon ou du cou la nuit dans l'enclos, ou sous des arbres devant la ferme.

Dans les élevages où les chèvreries sont modernes, les animaux sont bien abrités, l'entretien de ces parcs est plus ou moins régulier et les dépenses en fin de chaque campagne sont faibles pour leur entretien (Carl et Van den Burg, 2004). Ceci confirme les situations observées chez des éleveurs à Kolda $(96,2 \%)$ et à Fouta Djallon (100 \%) qui ont construit des chèvreries sur pilotis afin de faciliter la collecte du fumier et donc d'assurer une intégration agriculture et élevage (Missohou et al., 2000).

Pour certains éleveurs le rare recours aux interventions sanitaires a été semblable à celui signalé au Cameroun (Manjeli et al., 1994). Les principales raisons évoquées étaient la cherté des produits et des traitements, ainsi que la difficulté d'accès aux services vétérinaires, ou à l'insuffisance de compétences de certains aides vétérinaires, formés sur le tas par les vétérinaires installés dans les grandes villes, pour assurer les soins dans les milieux reculés. La PPR est aussi une contrainte au développement de l'élevage en RDC (Wasso et al., 2018). Les maladies rencontrées dans les élevages sont aussi enregistrées en station (Djagba et al., 2017). Le doute de l'efficacité de la vaccination pose le problème de la conservation du vaccin (la chaîne du froid) lors du transport du vaccin jusqu'au lieu de la vaccination. L'impact de la PPR comme l'une des principales causes de mortalités des caprins a été signalé en Afrique de l'Ouest par plusieurs auteurs (Traoré, 1985).

La complémentation alimentaire a été observée dans la majorité des élevages avec des sous-produits de récoltes et de transformation de ces récoltes (fanes d'arachides, drêche de boisson locale, déchets de cuisine). Cette pratique est rapportée par d'autres auteurs au Togo comme au Burkina (Guingouain, 2017 ; Gnanda et al., 2016). Au Nigeria, le faible apport d'aliment ou de fourrage aux caprins a été signalé par Lawal-Adebowale (2012). Certains éleveurs argumentent que les chèvres sont capables de vivre sur des espaces avec un pâturage rare, car elles peuvent profiter des pâturages aériens ou des débris végétaux (Zakara, 1985). D’autres disent que les caprins ne mangent pas beaucoup ou qu'ils sont moins sensibles au déficit alimentaire que les ovins (Missohou et al., 2016). Le niveau de complémentation alimentaire enregistré a été supérieur à celui signalé au Cameroun par Manjeli et al. (1994). Les aliments utilisés étaient aussi ceux utilisés au Burkina Faso (Tamboura et Berte, 1994). Aucune complémentation alimentaire à base de provende ou de graines de coton n'a été rapportée dans ces élevages contrairement au $7 \%$ des animaux qui bénéficient de l'apport de provende au Cameroun (Tchouamo et al., 2005) et aux caprins de la station de recherche de Kolokopé au Togo complémentés avec des graines de coton (Djagba et al., 2017). Au Sénégal, l'effet positif de la complémentation sur les paramètres de productivité des troupeaux est documenté (Tourrand et Landais, 1996). La rareté de distribution des pierres à lécher est signalée au Nord Togo aussi par Guingouain (2017). Ce taux est plus élevé que celui de 2,8 \% signalé au Burkina (Tamboura et Berte, 1994).

Dans la majorité des élevages visités, les animaux étaient sous surveillance pendant la saison des cultures (avril à novembre). La claustration a surtout été observée dans le sud du pays. Les animaux enfermés dans des enclos le plus souvent de fortune étaient nourris de 
feuilles, de branchages et de déchets de cuisine (Guingouain, 2017). Dans certains élevages, ils étaient libérés tôt le matin ou tard le soir pour pâturer autour des terrains d'école ou des maisons sur les terres laissées en jachère, et enfermés au retour à la ferme. Ce système a aussi été signalé au Nigeria (Lawal-Adebowale, 2012 ; Guingouain, 2017). L'attache au piquet est une méthode très pratiquée dans le nord du pays et dans le sud par certains éleveurs, immigrés des zones nord, à la recherche de terres pour les cultures. Cette observation a été faite aussi au nord du pays (Guingouain, 2017).

Les éleveurs expliquaient la non-conduite des caprins au pâturage, d'une part, par leur effectif faible dans les ménages et, d'autre part, par leur caractère difficile à conduire en groupe (préjugé sur la race Djallonké). Il a été constaté que dans certaines localités rurales dans la région des Plateaux, les animaux étaient en divagation totale. Dans ces villages, la communauté interdisait les cultures dans les cours des maisons afin de faciliter l'élevage des petits ruminants et des porcs.

Dans l'ensemble des élevages, en fin de saison des cultures, les caprins étaient en divagation et valorisaient ainsi les résidus de culture, les feuilles mortes des arbres et les repousses après les feux de brousse. Cette pratique est aussi signalée par Killanga et Traoré (1999) au Burkina Faso, et Guingouain (2017) au Nord Togo. La divagation totale des caprins est rapportée dans plus de $5 \%$ des élevages au Cameroun (Manjeli et al., 1994). La conduite des chèvres Sahéliennes au pâturage en groupe est une pratique courante (Ba Diao, 1991), et leur présence au sein d'un troupeau permet l'adaptation rapide des chèvres Djallonké présentes.

\section{- CONCLUSION}

Lélevage des caprins au Togo est basé sur la production de viande. La croissance démographique avec la réduction des jachères et le caractère grégaire de la chèvre Djallonké expliquent l'absence d'éleveurs entrepreneurs pour les caprins au Togo. Le non-entretien des abris des animaux, l'apport faible de compléments alimentaires et minéraux de qualité, le manque de soins vétérinaires appropriés et aussi le manque de boucs améliorateurs dans la plupart des élevages enquêtés sont les contraintes pour l'amélioration de la productivité de ces élevages. Aussi, le manque d'enregistrement des données (naissances, mortalités, ventes, pertes, dépenses pour soins ou achats d'intrants) ne permet pas le calcul de la productivité et/ou de la rentabilité.

La forte implication des jeunes et le taux élevé d'éleveurs instruits indiquent que l'élevage des caprins a de l'avenir au Togo. Il est donc nécessaire de procéder à une vaste campagne de sensibilisation et surtout de formation sur les bonnes pratiques d'élevage selon des itinéraires techniques adaptés aux conditions socioéconomiques et d'élevage des différentes régions. La détermination du calendrier de prophylaxie (surtout la lutte contre les parasites internes et externes) adapté aux différentes zones agroécologiques du pays est nécessaire. La promotion de la formation des associations d'éleveurs ou de plateforme élevage de caprins permettra de réduire certains frais de soins ou d'intrants alimentaires pour les animaux. Une mesure politique fixant un prix favorable du kilogramme de poids vif de l'animal à la vente est une piste en vue de la relance de la filière caprine.

\section{Remerciements}

Nous remercions le Programme de productivité agricole de l'Afrique de l'Ouest pour le Togo pour son soutien qui nous a permis de faire les enquêtes sur le terrain. Nos remerciements vont aussi au Dr Akantetou, directeur du Centre de recherche agronomique des savanes humides pour toutes les facilités qu'il nous a accordées lors de nos déplacements sur le terrain. Nous remercions également Z. Koffi, chercheur au Programme national coton, S. Boma, chercheur à
l'Institut togolais de recherche agronomique, M. Gamado, technicien au Programme national ovins-caprins, et les éleveurs et conseillers agricoles qui nous ont accompagnés pendant cette étude.

\section{Déclaration des contributions des auteurs}

AYD : conception, collecte des données, analyse et interprétation des données, et rédaction de la première version du manuscrit ; $\mathrm{BB}$ et AK : planification, révision critique du manuscrit et accord final de la version à publier; $\mathrm{HB}$ : planification, interprétation des résultats et révision critique du manuscrit ; $\mathrm{KN}$ : collecte des données, analyse et interprétation des données.

\section{REFERENCES}

Alexandre G., Arquet R., Fleury J., Troupé W., Boval M., Archimède H., Mahieu M., et al., 2012. Systèmes d'élevage caprins en zone tropicale : analyse des fonctions et des performances. In : Elevage caprin (eds Baumont R., Sauvant D.). Prod. Anim., 25 : 305-316

Ba Diao M., 1991. Les systèmes d'élevage dans la zone des Niayes au Sénégal. ISRA, Dakar, Sénégal (Etudes et documents ; 4)

Ba Diao M., Gueye A., Seck M., 1996. Facteurs de variation de la production laitière des caprins en milieu Peul. In: Proc. 3rd Biennial Conf. African Small Ruminant Research Network (Eds Lebbie S.H., Kagwini E.), Kampala, Uganda, 5-9 Dec. 1994. ILRI, Nairobi, Kenya, 117-129

Barry M.B., 1985. Le rôle des petits ruminants dans la stratégie d'intensification des systèmes de production en Côte d'Ivoire. In: Proc. Conf. Small Ruminants in African Agriculture (Eds. Wilson R.T., Bourzat D.), Addis Ababa, Ethiopia, 30 Sept.-4 Oct. 1985. ILCA, Addis Ababa, Ethiopia, 199206

Carl J., Van den Burg K., 2004. L'élevage de chèvres dans les zones tropicales. Digigrafi, Wageningen, Pays-Bas, 103 p.

Chetroiu R., Călin I., Niculescu G.C., 2013. Worldwide trends and orientations of raising goats. Munich Personal RePEc Archive Online, Germany (MPRA Paper 53460)

Djagba A.Y., Bonfoh B., Aklikokou K., Bassowa H., Koffi K.Z., 2017. Facteurs agissant sur la mortalité des chevreaux Djallonké et croisés Djallonké et Sahélien à la station de Kolokopé. Tropicultura, 35 (4) : 325-336

FAOstat, 2017. Statistical database, www.fao.org (consulté 25 mars 2018)

Gnanda B.I., Wereme N'Diaye A., Sanon H.O., Somda J., Nianogo J.A., 2016. Rôle et place des caprins dans les ménages au Sahel. Tropicultura, 34 (1) : 10-25

Guingouain C.H.G.L.N., 2017. L'élevage des petits ruminants en milieu paysan dans les régions de la Kara et des Savanes au Togo : Diagnostic technico-économique. Thèse Doct. Vét., Ecole nationale vétérinaire, MaisonsAlfort, France, 209 p.

Killanga S., Traore A., 1999. Influence de la gestion et du statut socio-économique de l'agropasteur sur la productivité des ovins et des caprins au Mali Central. Tropicultura (16-17) : 180-183

Landais E., Bonnemaire J., 1996. La zootechnie, art ou science ? Entre nature et société, l'histoire exemplaire d'une discipline finalisée. Courrier Environ. INRA, $27: 23-44$

Lawal-Adebowale O.A., 2012. Dynamics of ruminant livestock management in the context of the Nigerian agricultural system. In: Livestock Production. IntechOpen, London, UK, 61-80, doi: 10.5772/52923

Ligios S., Carta A., Bitti P.L., Tuveri I., 2004. Description des systèmes d'élevage caprin en Sardaigne et évaluation des stratégies d'amélioration génétique. In : L'évolution des systèmes de production ovine et caprine : avenir des systèmes extensifs face aux changements de la société (ed. Dubeuf J.P.). Options Méditer. Sér. A, (61) : 97-104

Manjeli Y., Teguia A., Njwe R.M. ; Tchoumboue J., Ayong E.E., 1994. Enquête sur l'élevage caprin dans les hauts plateaux de l'Ouest du Cameroun. In: Proc. 3rd Biennial Conf. African Small Ruminant Research Network (Eds Lebbie S.H., Kagwini E.), Kampala, Uganda, 5-9 Dec. 1994. ILRI, Nairobi, Kenya, 99-103

Missohou A., Ba A.C., Dieye P.N., Bah H., LO A., Gueye S., 2000. Ressources génétiques caprines d'Afrique de l'Ouest : systèmes d'élevage et caractères ethniques. In : XIle Conf. int. sur la chèvre, Tours, France, 20-24 mai 2000 
Missohou A., Nahimana G., Ayssiwede S.B., Sembène M., 2016. Goat breeding in West Africa: A review. Rev. Elev. Med. Vet. Pays Trop., 69 (1) : 3-18, doi : 10.19182/remvt.31167

Montfort A., 1990. Connaissance de la chèvre naine guinéenne et de son élevage : proposition d'amélioration en Côte d'Ivoire à partir des données disponibles pour les pays côtiers des zones humides et subhumides d'Afrique de I'Ouest. Mém. DAA, Institut national agronomique, Paris-Grignon, France, $58 \mathrm{p}$

Najari S., Gaddour A., 2008. Principaux systèmes d'élevage caprin et ovin des régions arides tunisiennes. Renc. Rech. Ruminants, 15 : 185

Nassif F., El Amiri B., Cohen N., 2011. L'élevage caprin à viande au Maroc : opportunités et perspectives (cas du caprin d'Ait Bazza). Bull. Transfert Technolo. Agric. (193)

Okello K.L., 1985. A survey of the productivity and functions of goats in Uganda. In: Proc. Conf. Small ruminants in African agriculture (Eds. Wilson R.T., Bourzat D.), Addis Ababa, Ethiopia, 30 Sept. - 4 Oct. 1985. ILCA, Addis Ababa, Ethiopia, 208-217

Otte M.J., Chilonda P., 2002. Cattle and small ruminant production systems in sub-Saharan Africa - a systematic review. FAO, Rome, Italy, 98 p.

République togolaise, 2018. Plan national de développement 2018-2022, www.republiquetogolaise.com/pnd, 158 p

Saidu O., Babafunso S., Galman O., Alaba A., Ishola M., 2006. West African Dwarf goat production under village conditions: 1 . Characterisation and the establishment of breed standards. In: Conf. Int. Agric. Res. Dev., Bonn, Germany, 11-13 Oct. 2006

Tamboura H., Berte D., 1994. Système d'élevage traditionnel caprin sur le Plateau Central du Burkina Faso In: Proc. 3rd Biennial Conf. African Small Ruminant Research Network (Eds Lebbie S.H., Kagwini E.), Kampala, Uganda, 5-9 Dec. 1994. ILRI, Nairobi, Kenya, 93-97

\section{Summary}

Djagba A.Y., Bonfoh B., Bassowa H., Aklikokou K., Kanour N. Assessment of goat rearing in a farming environment in Togo

Goats are the second most important livestock species after poultry in Togo. The availability of its meat is however rare. The Togolese State, through development projects, has initiated the recapitalization of the national short-cycle livestock. The objective of the study was to understand the weaknesses of goat-rearing practices in Togo with a view to proposing lines of research for its improvement and development. Surveys were thus conducted among 129 heads of households of both sexes. The breeders were farmers, employees or retired people. The surveys covered the entire country, taking into account its rainfall and plant diversity. Data on livestock practices, veterinary care, diseases and other constraints were collected. The results showed that $69 \%$ of the farmers were educated, $91 \%$ were married, and $26 \%$ of the owners were women. Family labor played an important role. Livestock activities were entrusted to women and children. The average household size was 18 goats. Adult goats made up $44 \%$ of the herd, compared to $2 \%$ of whole adult males. The weaning productivity rate was 0.54 kid per mother. The animals were provided with a shelter; in $85 \%$ of the farms they received periodic feed supplementation and in $27 \%$ of them a mineral supplement based on cooking salt. Veterinary care was observed in $64 \%$ of the farms. This study revealed some weaknesses in veterinary care, the use of improved sires and shelter availability. Training on technical itineraries for goat rearing is necessary to improve performance.

Keywords: goats, kids, West African Dwarf, productivity, Togo
Tchouamo I.R., Tchoumboue J., Lise T., 2005. Caractéristiques socio-économiques et techniques de l'élevage de petits ruminants dans la province de I'ouest du Cameroun. Tropicultura, 23 (4) : 201-211

Tourrand J.F., Landais E., 1996. Goat productivity in farming production systems of the Senegal River Delta. Rev. Elev. Med. Vet. Pays Trop., 49 (2) : 168-173, doi : 10.19182/remvt.9535

Traoré A., 1985. Causes de mortalités avant sevrage chez les ovins et caprins du système agropastoral du Mali central. In: Small Ruminants in Africa Agriculture (Ed. Wilson R.T., Bourzat D.). ILCA, Addis Ababa, Ethiopia, 119-128

Vanwarbeck O., 2008. Caractérisation technico-économique des élevages des chèvres laitières en Région de Wallonne. Bachelier Agronomie, option techniques et gestion agricoles, Haute Ecole de la Province de Liège, Belgique, $118 p$

Wasso D.S., Akilimali J.I., Patrick B., Bajope J.B., 2018. Elevage caprin : Situation actuelle, défis et impact socioéconomique sur la population du territoire de Walungu, République Démocratique du Congo. J. Appl. Biosci., 129 : 13050-13060, doi : 10.4314/jab.v129i1.8

Yandia M.C., 2012. Analyse du système de commercialisation des caprins dans la ville de Bangui (Centrafrique). Mém. Master Prod. Anim. Dév. Durable, Université Cheikh Anta Diop, Ecole inter-Etats des sciences et de médecine, Dakar, Sénégal, 32 p.

Zakara O., 1985. Les petits ruminants en République en Niger. In: Proc. Conf. Small ruminants in African agriculture (Eds. Wilson R.T., Bourzat D.), Addis Ababa, Ethiopia, 30 Sept. - 4 Oct. 1985. ILCA, Addis Ababa, Ethiopia, 236-242

\section{Resumen}

Djagba A.Y., Bonfoh B., Bassowa H., Aklikokou K., Kanour N. Evaluación de la situación de la cría de cabras en un medio campesino en Togo

En términos cuantitativos, las cabras constituyen la segunda especie de ganado en Togo después de las aves de corral. La disponibilidad de su carne sigue siendo rara. El Estado de Togo, a través de proyectos de desarrollo, ha iniciado el acrecentamiento del rebaño nacional de animales de ciclo corto. El objetivo del presente estudio fue conocer los puntos frágiles de las prácticas de cría de cabras en Togo, con el fin de proponer vías de investigación para su mejora y desarrollo. Se realizaron encuestas a 129 jefes de familia de ambos sexos. Los criadores eran campesinos, empleados o jubilados. Las encuestas se extendieron a todo el país, teniendo en cuenta la pluviosidad y la diversidad de plantas. Se recopilaron datos sobre prácticas de cría, atención veterinaria, enfermedades y otras limitaciones. Los resultados mostraron que el $69 \%$ de los criadores tenían educación, el 91\% estaban casados y el $26 \%$ de los propietarios eran mujeres. El trabajo familiar jugó un papel importante. Las actividades relacionadas con la cría de animales se confiaron a mujeres y niños. El tamaño promedio del rebaño por hogar fue de 18 cabras. Las cabras adultas constituyeron el $44 \%$ del hato, contra $2 \%$ de machos adultos enteros. La tasa de productividad al destete fue de 0,54 cabrito por hembra. Los animales tenían refugio; en el $85 \%$ de las fincas recibieron periódicamente un suplemento alimenticio y en el $27 \%$ de estas un suplemento mineral a base de sal de cocina. Se observó cuidado veterinario en el $64 \%$ de las fincas. Este estudio reveló algunas debilidades en la atención veterinaria, el uso de reproductores mejorados y la disponibilidad de refugio. La capacitación con protocolos técnicos de la cría de cabras es necesaria para mejorar su rendimiento.

Palabras clave: caprinos, cabrito, Djallonke, productividad, Togo 
